\title{
Using LoRaWAN smart city infrastructure as backup network for Industry 4.0 enabled vehicles
}

\author{
Paolo Ferrari $^{\S}$, Emiliano Sisinni ${ }^{\S}$, Diego Fernandes Carvalho ${ }^{\S}$, Gabriel Signoretti ${ }^{\dagger}$, Marianne Silva $^{\dagger}$, \\ Ivanovitch Silva ${ }^{\dagger}$ e Diego Silva ${ }^{\dagger}$ \\ $\S$ University of Brescia - Brescia, Italy \\ ${ }^{\dagger}$ Federal University of Rio Grande do Norte - Natal, Brazil \\ Emails: ${ }^{\S}$ \{paolo.ferrari, emiliano.sisinni, d.fernandescarva $\} @$ unibs.it, ${ }^{\dagger}\{$ gabrielsig, dinizmarianne $\} @$ ufrn.edu.br, \\ †ivan@imd.ufrn.br e ${ }^{\dagger}$ diego@ect.ufrn.br
}

\begin{abstract}
Intelligent vehicles are the very next future of the automotive sector, which is renewing itself following Industry 4.0 paradigms. Today, car manufacturers are rapidly increasing the number of vehicles that continuously transmit data to the Internet, in order to enable the virtuous feedback paths foreseen by Industry 4.0. Such data are used to improve production (and products) during the entire lifetime of plants (and cars, also). A data link between on board devices and Internet must be created. Currently, the On Board Diagnostic(OBD) interface is available in the majority of cars and trucks. Some Edge devices with OBD and 3G/4G modems have been created, enabling easy Internet connection. However, congestion situation, as well as temporary outages, may create significant holes in the 3G/4G coverage. Recently, the transformation of city into Smart City is begun. An impressive number of new sensors are deployed in urban area. New wireless technologies are on the rise and, among them, LoRaWAN is the most used. If a vehicle is considered like a "mobile sensor", then a viable option to fill 3G/4G gaps is the use of LoRaWAN infrastructure inside a Smart City. This paper deals with the creation of the prototype of an embedded platform which includes both OBD-II interface, 3G/4G connectivity and also LoRaWAN for backup situation. Considering the Smart City use cases, the application constrains regarding intelligent vehicles are discussed in order highlight design directions for the correct integration with the LoRaWAN infrastructure.
\end{abstract}

Index Terms-Internet of Intelligent Vehicles, OBD-II, Edge Computing, Low Power WAN, Wireless sensor networks

\section{INTRODUÇÃO}

O cenário atual de mobilidade está mudando rapidamente e a definição de veículos está se tornando mais ampla. Por exemplo, veículos híbridos/completamente elétricos, motocicletas leves e sistemas autônomos podem ser encontrados em muitas cidades hoje [1]. Para atualizar o mercado de veículos, tornando-o sustentável e lucrativo (novamente), os fabricantes estão investindo na aplicação do paradigma Industria 4.0. Esse objetivo exige ter veículos que transmitam dados continuamente para a Internet, permitindo um feedback contínuo do "produto" como previsto pela Industria 4.0. Os fabricantes de automóveis estão usando esses dados para melhorar a qualidade da produção, diminuindo os erros de projeto e aumentando a flexibilidade da fábrica [2].

No entanto, o fluxo de dados provenientes dos veículos que podem se estender por toda a sua vida útil, podem ser úteis para outras aplicações. $\mathrm{O}$ uso cruzado de dados para obter informações sobre o ambiente ao redor dos veículos é uma das ideias principais por trás da Internet de Veículos Inteligentes (IoIV). Nesta visão, os veículos estão cheios de sensores e podem ser vistos como sondas imersas no ambiente; qualquer serviço utilizando as medições provenientes de fontes de dados de veículos poderia ser ativado [3], [4]. Em particular, as aplicações mais eficazes são possíveis quando a própria cidade faz parte da estrutura inteligente. $\mathrm{O}$ conceito de cidade inteligente é o modelo de referência para cidades que evoluem para o futuro. Na Cidade Inteligente, o papel da Internet como tecido interconectado é crucial. Os cidadãos e os veículos são partes essenciais da cidade inteligente, juntamente com edifícios e infraestruturas inteligentes [5].

Não apenas novos modelos de carros podem fazer parte dessa revolução da IoIV; qualquer veículo recente pode ser equipado com dispositivos de comunicação que extraem informações dos sensores a bordo. De fato, a interface $\mathrm{On}$ Board Diagnostic (OBD) existe na maioria dos carros e caminhões. Existem também dispositivos com modems OBD e 3G/4G com o objetivo específico de estabelecer conexões com a Internet. O principal obstáculo para a integração perfeita é atualmente o congestionamento de trafego de dados, bem como as interrupções temporárias da rede $3 \mathrm{G} / 4 \mathrm{G}$ que podem afetar o fluxo contínuo de informações.

Este trabalho propõe explorar oportunisticamente a infraestrutura das Cidades Inteligentes como um caminho de backup para informações provenientes de veículos. Em especial, as tecnologias Low Power Wide Area Network (LPWAN) onde destaca-se o uso do LoRaWAN. Na Seção III deste documento, é apresentado um protótipo de uma plataforma incorporada que inclui interface OBD-II, conectividade 3G/4G e LoRaWAN para situações de backup. Em seguida, na Seção IV, é dado um exemplo de Cidade Inteligente inovadora com infraestrutura LoRaWAN. Por fim, na Seção V, são discutidas as restrições da solução (em termos de alcance e confiabilidade) no contexto de casos de uso de Cidades Inteligentes assim como um conjunto de boas práticas para utilização da infraestrutura LoRaWAN.

\section{Visão Geral da Tecnologia e Objetivos}

Os paradigmas da Internet das Coisas, Indústria 4.0 e veículos inteligentes estão impulsionando a introdução de várias novas tecnologias. A seguir é apresentada uma breve 
visão geral das tecnologias relevantes para este trabalho, seguida de uma análise do atual estado da arte e, por fim, a lista dos objetivos do trabalho.

\section{A. $O B D-I I$}

O OBD-II é um dispositivo conectado à Unidade de Controle do Motor (ECU, do Inglês Engine Control Unit veículo. Ele fornece uma interface de comunicação para a troca de dados fornecidos em tempo real pelos sensores e atuadores do veículo [6]. A comunicação entre o OBD-II e a ECU do carro é possível por meio de alguns protocolos diferentes. Normalmente, cada veículo carrega apenas um protocolo e o mais utilizado é o Controller Area Network (CAN), desenvolvido pela Bosh em 1980. No entanto, também existem outros protocolos como o SAE J1850 PWM, SAE J1850 VPW, ISO 9141-2.

A interface OBD-II possui dez modos de operação para cumprir propósitos específicos. Existem conjuntos distintos de comandos usados para extrair dados de sensores e atuadores dos veículos [7], usando códigos exclusivos conhecidos como Parameter IDs (PIDs). Não é obrigatório que um fabricante implemente todas as funcionalidades em todos os seus veículos. Portanto, é importante verificar quais comandos o veículo suporta antes das interações.

O sistema de aquisição e análise de dados considerado neste documento é ligado à porta OBD-II do veículo e configurado para enviar os dados armazenados para o banco de dados na cloud computing por meio da conexão de rede disponível "atualmente".

\section{B. LoRaWAN}

As aplicações no cenário de IoT exigem baixo custo, escalabilidade e uma grande cobertura com baixo consumo de energia. As tecnologias WAN de baixa potência (LPWAN) atendem a essas necessidades quando uma baixa taxa de dados pode ser tolerada. Geralmente, as redes sem fio LPWAN possuem os seguintes recursos: operam em bandas de frequência diferentes da frequência ISM de 2,4 GHz; oferecem baixa taxa de transmissão de dados; possuem um esquema de modulação eficiente e uma excelente sensibilidade devido à pequena largura de banda do canal (ganhos e perdas do transmissor é da ordem de $150 \mathrm{~dB}$ ); protocolo e infraestrutura de comunicação simplificados (resultando em muito baixo consumo de energia e redução de custos).

As redes LPWAN contam com topologia em estrela de salto único; o acesso ao meio é um mecanismo aleatório (por exemplo, ALOHA [8]), enquanto os regulamentos regionais frequentemente exigem um controle rigoroso sobre a transmissão do ciclo de trabalho. Geralmente, a complexidade extremamente baixa dos dispositivos finais é contrabalançada pela alta complexidade do sistema de back-end.

Entre as diferentes tecnologias da família LPWAN [9], a mais interessante e conhecida é a LoRaWAN, que explora a modulação Chirp Spread Spectrum (CSS) dos rádios LoRa, operando nas bandas sub-GHz sem licença.
A especificação LoRaWAN abrange a camada de enlace de dados, incluindo uma estratégia de acesso ao meio semelhante ao ALOHA. A comunicação sem fio LoRaWAN é a última milha de uma rede híbrida sem fio e com fio, organizada em uma topologia em estrela. Esta técnica vem da topologia de redes móveis (celulares), na qual os dispositivos formam uma estrela sem fio com várias estações base encaminhando dados para um controlador de estação base por meio de conexões com fio [10]. O LoRaWAN define gateways (executando o software de encaminhamento de pacotes) na fronteira entre redes sem fio e com fio: eles apenas encaminham mensagens de dispositivos finais para os servidores back-end. O gateway funciona na camada física e a carga útil do quadro é transparente para ele. De fato, existem duas camadas no LoRaWAN: a primeira utiliza as transmissões sem fio e fornece conectividade aos dispositivos finais; a segunda é o back-end que controla a rede de maneira centralizada e oferece serviços aos usuários finais.

Existem dois tipos de troca de dados pela rede LoRaWAN: "uplink" (comunicação do dispositivo final com os servidores back-end) e "downlink" (da maneira oposta). De acordo com o design do LoRaWAN, o "uplink" é o mais importante e usado, enquanto as mensagens de "downlink" são pouco transmitidas, pois podem diminuir a utilização da largura de banda [11].

As especificações LoRaWAN [12] descrevem um modelo de referência de rede incluindo três servidores: o Network Server (NS); o Application Server (AS) e o Join Server (JS).

O NS constitui o núcleo da topologia em estrela lógica. O NS examina o formato de quadro correto e é responsável pela autenticação de rede de forma consistente; também fornece confirmações para mensagens que precisam de confirmação. Além disso, o NS gerencia qualquer funcionalidade LoRaWAN, como a adaptação da taxa de dados, por exemplo. O NS encaminha as mensagens de "uplink" dos dispositivos finais para o AS, enquanto, na direção inversa, enfileira o fluxo de "downlink" de qualquer AS para o dispositivo final correto. Quando um JS é colocado, o NS é responsável por trocar as mensagens "Join-request" e "Join-accept" entre os dispositivos finais e o JS. Por fim, o AS envia dados da aplicação aos usuários finais usando protocolos conhecidos (por exemplo, com base em HTTP, REST ou MQTT), porque esse detalhe não está descrito nas especificações.

\section{Trabalhos relacionados e objetivos}

A literatura científica sobre a IoIV, o uso do LoRaWAN para veículos e a inclusão de veículos no ecossistema das Cidades Inteligentes está aumentando. Um sistema de gestão de frota baseado no OBD-II é realizado em [13]. Um leitor OBD-II é usado para coletar dados de velocidade e MAF, a partir dos quais a distância percorrida e o consumo de combustível também são medidos. Nesse mesmo caminho, uma plataforma de feedback do cliente para a indústria automotiva no cenário da Indústria 4.0 é ilustrada em [7]. Essa plataforma é capaz de obter e examinar dados dos sensores do veículo a fim de gerenciar o controle, a prevenção e a mitigação dos problemas do carro. O sistema transmite as mensagens coletadas por 
um leitor OBD-II. Além disso, [14] sugeriram uma solução de banco de dados de gráficos (orientada para a Indústria 4.0) para gerenciar a enorme quantidade de dados criados pelos sensores espalhados dentro do veículo em um cenário de IoT. Alguns benefícios foram reconhecidos por meio da localização geográfica dos veículos e eventos descobertos. Outras soluções poderiam também ser exploradas, como é o caso do monitoramento de poluição [15].

Os veículos elétricos serão uma parte essencial dos futuros cenários de mobilidade urbana dentro das Cidades Inteligentes. Em [16] é relatada uma análise detalhada dos requisitos de comunicação para o gerenciamento de estações de recarga; 4G e LoRaWAN são considerados e comparados.

A cobertura de 3G/4G dentro da área urbana pode ser imprevisível. Em [17], alguns dos autores analisaram a situação atual em uma grande cidade do Brasil, encontrando evidências de falta de cobertura quando dispositivos $3 \mathrm{G}$ estão a bordo de veículos em movimento (em condições reais de tráfego).

Por fim, a cobertura de áreas extensas por meio da infraestrutura LPWAN é uma realidade em várias cidades. Por exemplo, a cidade de Brescia (Itália) possui uma das mais completas infraestruturas LoRaWAN da Europa, conforme descrito nesses trabalhos [18], [19].

Com base na literatura anterior, o principal objetivo deste trabalho é fornecer uma solução mais flexível, baseada em OBD-II, para monitoramento de veículos orientada para aplicações da Indústria 4.0. A nova arquitetura deve levar em conta e usar oportunamente todos os canais de comunicação disponíveis nas Cidades Inteligentes modernas, a fim de garantir o fluxo constante de informações. Em particular, a tecnologia LoRaWAN foi avaliada como a solução mais viável.

\section{PRotótipo Proposto}

O protótipo proposto explora uma rede de backup adequada para superar a possível falta de cobertura da infraestrutura 3G/4G. Os objetivos do projeto são:

- Longo alcance

- Capacidade de transmissão de dados obrigatórios para garantir o monitoramento contínuo das atividades de veículos/motoristas

- Transmissão em tempo real com latência reduzida

A arquitetura proposta é baseada em uma plataforma previamente avaliada na Universidade Federal do Rio Grande do Norte (UFRN) [17], combinada com a capacidade de longo alcance do protocolo LoRaWAN. Em detalhes, a arquitetura é mostrada na Figura 1. Os dados provenientes da interface OBD II são lidos usando um dispositivo AutoPi Edge com capacidade OBD II. A transmissão LoRaWAN é obtida por meio de um modem LoRaWAN conectado ao dispositivo.

\section{A. Protótipo proposto}

O dispositivo AutoPi é um verdadeiro dispositivo de computação Edge, pois é equipado com um módulo Raspberry Pi Zero (CPU de núcleo único de $1 \mathrm{GHz}, 512 \mathrm{MB}$ de RAM). O dispositivo está executando uma versão modificada do Raspbian Linux. A interface LoRaWAN foi implementada com

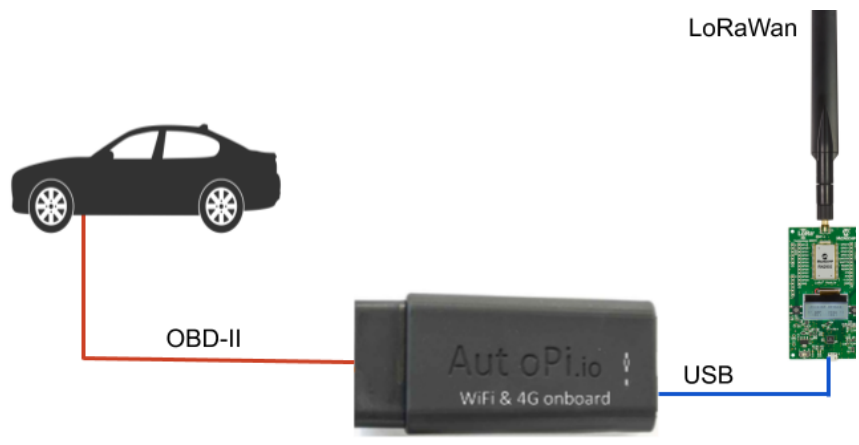

Fig. 1. Proposta de integração de módulos para transmissão LoRaWAN como backup para WiFi e 3G/4G padrão.

um software Python com ferramenta serial mini-term conectada por meio de uma porta USB ao Microchip LoRaWAN Module RN2903 da Microchip (operando na banda ISM de $915 \mathrm{MHz}$ ) com pilha LoRaWAN 1.0.1. (Nota: O módulo Microchip RN2483 também pode ser usado se o protótipo estiver operando na banda europeia em $868 \mathrm{MHz}$ ).

A parte mais crítica do design do protótipo foi a definição dos dados a serem transmitidos por meio do LoRaWAN, uma vez que existem limitações regionais de tamanho de pacote e limitações de ciclo de trabalho, conforme indicado anteriormente.

Os dados dentro do veículo são agrupados por PID, conforme discutido anteriormente. O tamanho de cada PID é bastante variável e muitas vezes inclui o registro de data e hora. Os PIDs mais importantes são: latitude, longitude, velocidade, Rotação por Minuto (RPM), posição do acelerador, acelerômetro, Fluxo de Ar em Massa (MAF) e Pressão do Ar no Coletor (MAP); com esses PIDs é possível monitorar as emissões veiculares e o comportamento do motorista. A Tabela I lista o tamanho em bytes (B) desses PIDs. O tamanho da carga útil das mensagens LoRaWAN está variando com a codificação SF, mas, para empacotar o máximo de informações possível, apenas os maiores pacotes LoRaWAN podem ser usados. Em particular, a carga útil transmitida tem 159B de comprimento (incluindo apenas o registro de data e hora da Latitude), permitindo que apenas SF7 e SF8 sejam usados. A

TABLE I

DETALHES DOS PIDS TRANSMITIDOS DENTRO DO Gateway LORAWAN. (TODOS OS TAMANHOS ESTÃO EM BYTES)

\begin{tabular}{c|c}
\hline PID & Tamanho \\
\hline Latitude & 12 \\
Longitude & 10 \\
Latitude e Longitude & 20 \\
Velocidade & 5 \\
RPM & 6 \\
Posição do Acelerador & 20 \\
MAF & 10 \\
MAP & 10 \\
Acelerômetro & 34 \\
Data e hora & 32 \\
\hline
\end{tabular}

carga útil máxima e as limitações do ciclo de trabalho variam 
de país para país. O ciclo de trabalho mais restritivo de $1 \%$ é considerado neste documento para maximizar a compatibilidade; pelo mesmo motivo, SF7 e SF8 @ BW $=125 \mathrm{KHz}$ foram abordados. Como o pacote LoRaWAN com carga útil de 160 bytes possui um tempo no ar de 262ms@SF7 e 462ms@SF8, a taxa máxima de atualização com uma conexão LoRaWAN de backup é de $26 \mathrm{~s}$ e $46 \mathrm{~s}$ (satisfazendo os requisitos do intervalo de 20 s entre sucessivos uplinks na regulamentação brasileira). Essa situação está muito longe do habitual caso $3 \mathrm{G} / 4 \mathrm{G}$, em que a taxa de atualização é da ordem de alguns segundos.

\section{INFraestrutura LoRAWAN EM CIDAdeS INTELIGENTES}

Nesta seção, é apresentado um exemplo de infraestrutura LoRaWAN real instalada em uma cidade inteligente. A cidade de Brescia (Itália) instalou nos últimos três anos uma das redes urbanas mais amplas baseadas na tecnologia LoRaWAN. A infraestrutura LoRaWAN é usada para lidar com serviços primários, como por exemplo: aquecimento urbano, coleta e manuseio de resíduos, monitoramento do consumo de água/energia (especialmente no campus inteligente da universidade [18]). Até a data atual, 123 Gateways LoRaWAN foram instalados em uma área densamente urbanizada de mais de $75 \mathrm{~km} 2$, resultando em uma densidade média de 1,6 Gateways $/ \mathrm{km} 2$. A distribuição geográfica dos gateways implantados é demonstrada na Figura 2, onde é visível que a densidade não é uniforme, porque a densidade do gateway também é proporcional à densidade dos nós finais nos arredores. Portanto, a maioria das áreas preenchidas contém mais nós finais, exigindo, por sua vez, mais gateways. Para avaliar o raio mínimo da célula coberta por um gateway, a distância mínima entre um gateway e seu gateway vizinho foi calculada. Na Figura 3 observa-se a distribuição das distâncias mínimas, destacando que a maioria dos gateways está localizada a cerca de $400 \mathrm{~m}$ do mais próximo. A distância média é de $500 \mathrm{~m}$, a distância mínima é de $110 \mathrm{~m}$ e a distância máxima é de $1500 \mathrm{~m}$.

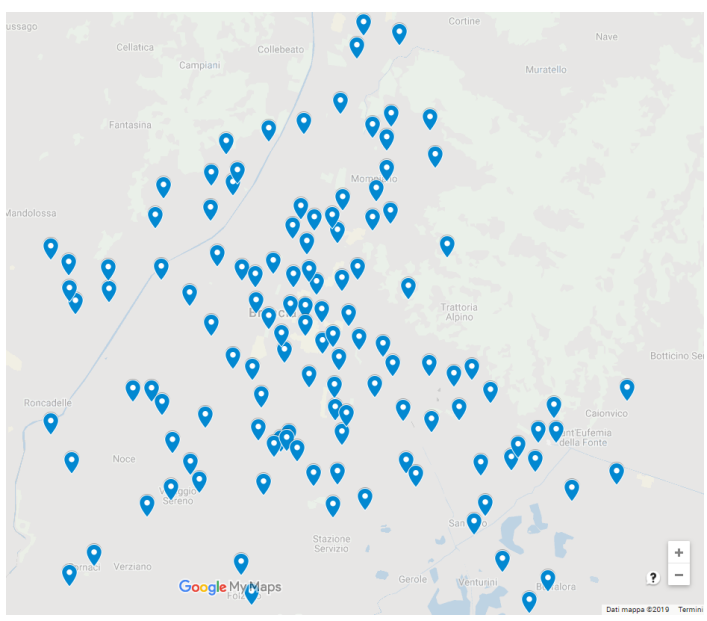

Fig. 2. Distribuição geográfica do Gateway LoRaWAN na cidade de Brescia.

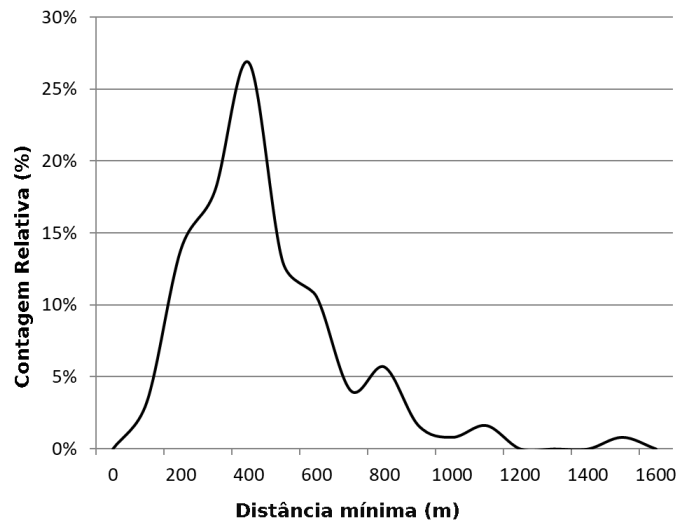

Fig. 3. Distribuição da distância mínima entre Gateway LoRaWAN na cidade de Brescia.

\section{Casos de Usos em Cidades Inteligentes}

Nesta seção, as restrições derivadas de casos de uso no cenário Cidades Inteligentes são introduzidas. Em particular, os experimentos são orientados para: avaliar a probabilidade de um nó final ser capaz de enviar seus dados para a infraestrutura considerada; e a distância entre o nó e a estação base para obter uma transmissão bem-sucedida. Em seguida, são fornecidas algumas instruções de design para a integração adequada de veículos inteligentes com recursos oportunistas de LoRaWAN.

\section{A. Caracterizando a cobertura $4 G$}

A caracterização da cobertura $4 \mathrm{G}$ foi realizada com experimentos realizados em uma rota urbana na cidade de Natal, Brasil. O percurso tem aproximadamente $5 \mathrm{~km}$ de extensão e foi executado em dois dias diferentes, das $9 \mathrm{~h}$ às $10 \mathrm{~h}$. Para cada dia do experimento, quatro rodadas foram concluídas com os carros listados na Tabela II. O experimento foi realizado nesses horários para obter maior variabilidade nos dados. Cada veículo foi equipado com o protótipo descrito na seção anterior. No dispositivo AutoPi, os parâmetros foram definidos da seguinte forma: tipo de conexão para $4 \mathrm{G}$, com APN Tim; servidor de recebimento de dados hospedado no AutoPi Web Service; Intervalo de solicitação de conjunto de PIDs configurado para 1s. Dessa forma, os dados foram coletados totalizando cerca de $20 \mathrm{~km}, 5 \mathrm{~km}$ em cada rodada do experimento por veículo. Os dados foram coletados em estradas urbanas, com duração total de 40 minutos e média de cerca de 10 minutos por carro. A visualização da rota é mostrada na Figura 4, onde os pontos representam as transmissões bem-sucedidas realizadas pela interface AutoPi $4 \mathrm{G}$.

TABLE II

Carros Utilizados nos Cenários Urbanos CONSIDERAdos NA CIDADE DE NATAL (BRASIL)

\begin{tabular}{c|c|c|c}
\hline Modelo & Motor & Transmissão & Ano \\
\hline Ford Ka & 1.5 & Automático & 2019 \\
Chevrolet Onix & 1.4 & Automático & 2015 \\
\hline
\end{tabular}




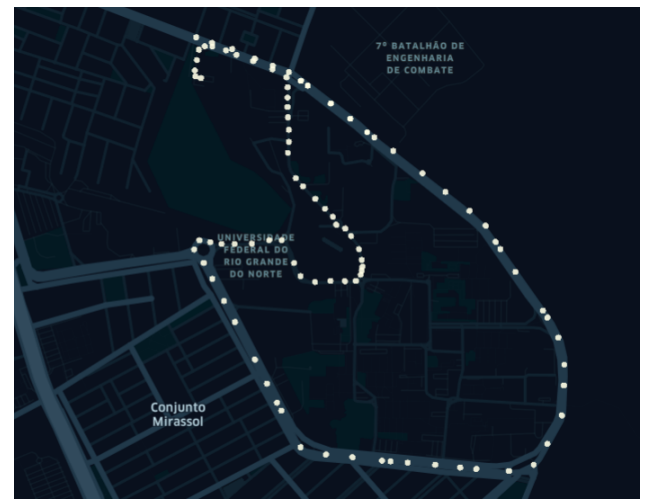

(a) Ford Ka.

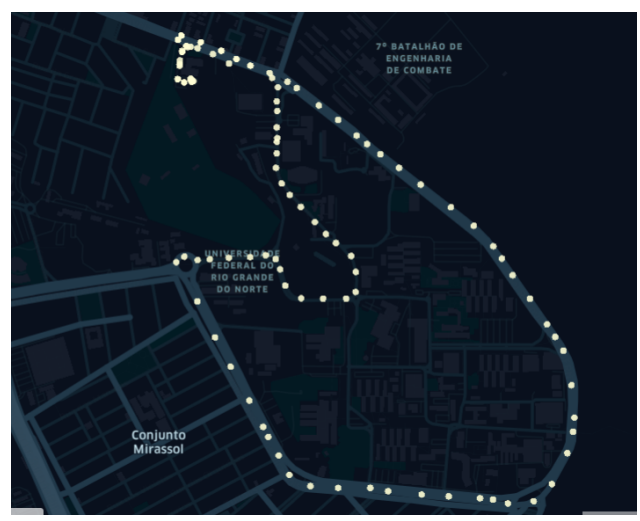

(b) Chevrolet Onix.

Fig. 4. Rota para o cenário urbano na Cidade de Natal.

Os resultados dos experimentos são mostrados na Tabela III. A taxa de falhas (número de tentativas com falha sobre o número total de tentativas de conexão) é da ordem de $10 \%$ quando o carro está em movimento.

TABLE III

TAXA DE FALHA NA CONEXÃO 4G NOS CENÁRIOS URBANOS CONSIDERADOS NA CIDADE DE NATAL (BRASIL)

\begin{tabular}{c|c|c|c|c}
\hline Modelo & Amostras & Falhas & \% Falha & \% Sucesso \\
\hline ka - dia 1 & 109 & 10 & 9 & 91 \\
ka - dia 2 & 120 & 11 & 9 & 91 \\
Onix - dia 1 & 106 & 11 & 11 & 89 \\
Onix - dia 2 & 120 & 16 & 13 & 87 \\
\hline
\end{tabular}

\section{B. Caracterização da cobertura LoRaWAN nas Cidades In- teligentes}

A cobertura do LoRaWAN no caso de nós finais fixos foi avaliada experimentalmente em Natal (Brasil). O caso dos nós móveis foi testado em Brescia (Itália).

1) Nó final fixo: A configuração experimental é composta por um gateway LoRaWAN, colocado no Laboratório de Informática Industrial da UFRN, e um nó final LoRaWAN, atuando como um sensor. O gateway LoRaWAN é um dispositivo Sentrius RG191 com as seguintes características: duas antenas WiFi de banda dupla, uma antena LoRa e uma porta Ethernet (RJ45). O gateway pode ter interface com muitas implementações de back-end do LoRaWAN: Semtech, LoRaIO, The Things Network (TTN), Stream etc. O nó final do LoRaWAN é realizado usando um módulo Microchip RN2903 que possui as seguintes características: Implementação da classe A LoRaWAN, suporte à banda ISM de $915 \mathrm{MHz}$ e à interface do modem ASCII.

O dispositivo final LoRaWAN é colocado em 20 pontos dentro do campus e envia pacotes contendo uma carga útil de 50B. A pior condição do ponto de vista da sensibilidade foi escolhida: as mensagens foram enviadas usando SF7 @ $\mathrm{C} / \mathrm{R}=4 / 5$. A potência de transmissão foi ajustada para $+14 \mathrm{dBm}$. Os locais testados foram escolhidos aumentando a distância entre o gateway e o mote LoRaWAN. A Figura 5 demonstra os locais do mote, identificados por marcas azuis (se a transmissão foi bem-sucedida) ou marcas vermelhas (quando a transmissão não foi bem-sucedida), bem como a posição do Gateway, identificada pela cruz roxa. Em cada local escolhido, o procedimento de teste foi repetido cinquenta vezes.

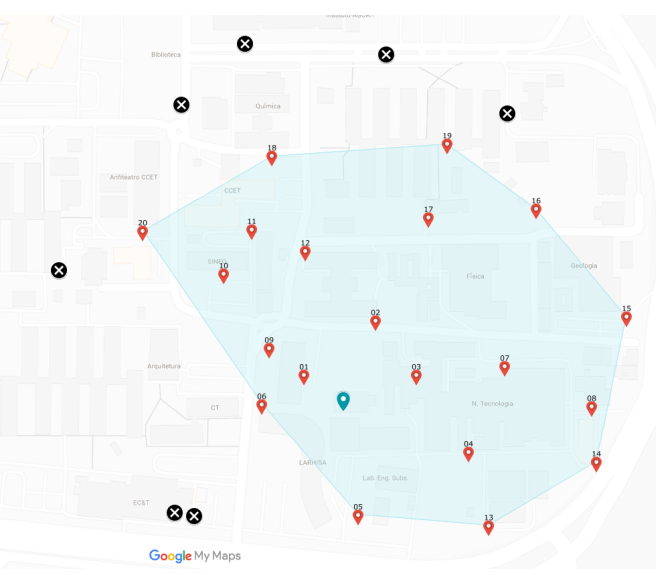

Fig. 5. Distribuição geográfica dos locais cobertos por um Gateway LoRaWAN no campus da UFRN na cidade de Natal.

A distância real de operação se mostrou ser até $250 \mathrm{~m}$ do gateway, quando esse cenário urbano é considerado (e o LoRaWAN opera com o código SF7 mais fraco). A presença de edifícios altos e terrenos complexos explica a área de cobertura parcialmente reduzida.

2) Nó final móvel: Cenários de mobilidade já foram avaliados para LoRaWAN dentro do projeto Brescia Smart Living [19]. O gateway LoRaWAN é o dispositivo Sentrius RG186, enquanto o nó final LoRaWAN é construído com um módulo Microchip RN2483. Um carro equipado com o transmissor LoRaWAN estava percorrendo um itinerário de cerca de $4 \mathrm{~km}$ à uma velocidade máxima de $50 \mathrm{~km} / \mathrm{h}$ e uma velocidade média de $17 \mathrm{~km} / \mathrm{h}$. Todos os pacotes enviados foram capturados por gateways pertencentes à infraestrutura da Cidade Inteligente descrita na seção anterior, confirmando a usabilidade do LoRaWAN para aplicativos móveis urbanos.

\section{Instrução de Revisão}

Os resultados discutidos anteriormente confirmam a viabilidade da abordagem proposta. Em particular, os experimentos 
permitiram identificar alguns pontos-chave.

Deve-se ressaltar que a necessidade de transferir uma quantidade relativamente grande de dados do usuário (pelo menos se comparada com o tamanho da mensagem dos aplicativos pretendidos para LoRaWAN, como medição inteligente) complementada por uma rápida taxa de atualização para garantir o comportamento em tempo real, força a adoção do taxas de dados mais rápidas, mas com menos ruído/interferência, suportadas pelo LoRaWAN. Por sua vez, isso implica que, quando um cenário urbano é considerado, o nó (móvel) deve ter uma distância do gateway mais próximo da ordem de 250 a 300m quando estiver trabalhando com o SF7. Esses tipos de implantações podem ser considerados relativamente densos, mas já foram realizados em muitos locais. A densidade de implantação sugerida é de cerca de 1,5 Gateway/km2.

Além disso, as limitações na transmissão de mensagens sucessivas afetam fortemente a taxa de atualização. Considerando o uso de SF7 e SF8@BW $=125 \mathrm{kHz}$ e d $=1 \%$ (permitido nas regiões da Europa e do Brasil e permitindo a carga útil de 160B desejada), a taxa de atualização sugerida é da ordem de 30 s e 60 s, respectivamente. Deve-se notar que em outros países um tempo máximo de permanência (ou um intervalo mínimo diferente entre uplinks sucessivos) pode ser especificado. No entanto, uma atualização mais lenta que o cenário $4 \mathrm{G}$ deve ser levada em consideração e gerenciada no nível da aplicação.

\section{CONCLUSÃO}

Veículos inteligentes são o futuro do setor automotivo, construído sobre paradigmas da Indústria 4.0. Os fabricantes de automóveis já estão coletando dados de veículos conectados à Internet para melhorar a produção (e produtos) durante toda a vida útil das fábricas (e produtos). Hoje a interface OBD-II é o ponto de acesso (para sensores a bordo) em muitos carros e caminhões. Para aumentar o número de veículos conectados podem ser utilizados dispositivos com modems OBD-II e 3G/4G, mesmo se houver situações em que a cobertura/capacidade $3 \mathrm{G} / 4 \mathrm{G}$ não seja suficiente. A ideia original deste artigo é explorar a infraestrutura da Cidade Inteligente como forma de conexão de backup para veículos inteligentes que perdem temporariamente seu principal link 3G/4G. As Cidades Inteligentes são fortemente baseadas no compartilhamento de dados e, para isso, uma infraestrutura de comunicação específica deve ser implantada. Em particular, novas tecnologias sem fio são amplamente utilizadas, como por exemplo LoRaWAN. Este artigo apresentou um protótipo de uma plataforma incorporada que inclui interface OBD-II, conectividade $3 \mathrm{G} / 4 \mathrm{G}$ e também conexão LoRaWAN oportunista para situações de backup. $\mathrm{O}$ trabalho considerou casos de uso de Cidades Inteligentes em relação a veículos inteligentes, derivando as restrições de aplicativos que podem ser usadas para a correta integração LoRaWAN.

\section{ACKNOWLEDGMENT}

Os autores gostariam de agradecer ao Ing. P. Palazzoli e L. Groppelli, da A2A Smart City, pela ajuda valiosa durante a discussão dos casos de uso.

\section{REFERÊNCIAS}

[1] C. Benevolo, R. P. Dameri, and B. D'Auria, "Smart mobility in smart city," in Empowering Organizations, pp. 13-28, Springer, 2016.

[2] R. Sugayama and E. Negrelli, "Connected vehicle on the way of industry 4.0," Available online: pdf. blucher. com. br. s3-sa-east-1. amazonaws. com/engineeringproceedings/simea2016/PAP16. pdf (accessed on 20 June 2018), 2015.

[3] W.-C. Wu and H.-T. Liaw, "The next generation of internet of things: Internet of vehicles," in International Conference on Frontier Computing, pp. 278-282, Springer, 2017.

[4] T. T. Dandala, V. Krishnamurthy, and R. Alwan, "Internet of vehicles (iov) for traffic management," in 2017 International Conference on Computer, Communication and Signal Processing (ICCCSP), pp. 1-4, Jan 2017.

[5] A. Zanella, N. Bui, A. Castellani, L. Vangelista, and M. Zorzi, "Internet of things for smart cities," IEEE Internet of Things journal, vol. 1, no. 1, pp. 22-32, 2014.

[6] M. Da Silva, E. Vieira, I. Silva, D. Silva, P. Ferrari, S. Rinaldi, and D. Carvalho, "A customer feedback platform for vehicle manufacturing in industry 4.0," vol. 2018-June, pp. 1249-1254, 2018.

[7] M. Silva, E. Vieira, G. Signoretti, I. Silva, D. Silva, and P. Ferrari, "A customer feedback platform for vehicle manufacturing compliant with industry 4.0 vision," Sensors, vol. 18, no. 10, 2018.

[8] D. Bankov, E. Khorov, and A. Lyakhov, "Mathematical model of lorawan channel access," in 2017 IEEE 18th International Symposium on A World of Wireless, Mobile and Multimedia Networks (WoWMoM), pp. 1-3, IEEE, 2017

[9] U. Raza, P. Kulkarni, and M. Sooriyabandara, "Low power wide area networks: An overview," IEEE Communications Surveys \& Tutorials, vol. 19, no. 2, pp. 855-873, 2017.

[10] M. Bauer, P. Bosch, N. Khrais, L. G. Samuel, and P. Schefczik, "The umts base station router," Bell Labs Technical Journal, vol. 11, no. 4, pp. 93-111, 2007.

[11] K. Mikhaylov, J. Petäjäjärvi, and A. Pouttu, "Effect of downlink traffic on performance of lorawan lpwa networks: Empirical study," in 2018 IEEE 29th Annual International Symposium on Personal, Indoor and Mobile Radio Communications (PIMRC), pp. 1-6, IEEE, 2018.

[12] "LoRaWAN® Specification v1.1 - LoRa Alliance®."

[13] R. Malekian, N. R. Moloisane, L. Nair, B. Maharaj, and U. A. ChudeOkonkwo, "Design and implementation of a wireless obd ii fleet management system," arXiv preprint arXiv:1701.02160, 2017.

[14] A. Pieroni, N. Scarpato, and M. Brilli, "Industry 4.0 revolution in autonomous and connected vehicle a now-conventional approach to manage big data.," Journal of Theoretical \& Applied Information Technology, vol. 96, no. 1, 2018.

[15] M. Silva, G. Signoretti, J. Oliveira, I. Silva, and D. G. Costa, "A crowdsensing platform for monitoring of vehicular emissions: A smart city perspective," Future Internet, vol. 11, no. 1, 2019.

[16] S. Rinaldi, M. Pasetti, E. Sisinni, F. Bonafini, P. Ferrari, M. Rizzi, and A. Flammini, "On the mobile communication requirements for the demand-side management of electric vehicles," Energies, vol. 11, no. 5, p. 1220, 2018.

[17] G. Signoretti, M. Silva, A. Dias, I. Silva, D. Silva, and P. Ferrari, "Performance evaluation of an edge obd-ii device for industry 4.0," in 2019 II Workshop on Metrology for Industry 4.0 and IoT (MetroInd4. O\&IoT), pp. 432-437, IEEE, 2019.

[18] F. Bonafini, D. F. Carvalho, A. Depari, P. Ferrari, A. Flammini, M. Pasetti, S. Rinaldi, and E. Sisinni, "Evaluating indoor and outdoor localization services for lorawan in smart city applications," in 2019 II Workshop on Metrology for Industry 4.0 and IoT (MetroInd4. O\&IoT), pp. 300-305, IEEE, 2019.

[19] D. F. Carvalho, A. Depari, P. Ferrari, A. Flammini, S. Rinaldi, and E. Sisinni, "On the feasibility of mobile sensing and tracking applications based on lpwan," in 2018 IEEE Sensors Applications Symposium (SAS), pp. 1-6, IEEE, 2018. 